\title{
ON THE RANK AND THE CRANK MODULO 4
}

\author{
RICHARD LEWIS
}

(Communicated by William Adams)

\begin{abstract}
Let $N(r, m, n)$ (respectively, $M(r, m, n)$ ) denote the number of partitions of $n$ whose ranks (respectively, cranks) are congruent to $r$ modulo $n$. It is shown that $N(0,4,2 n+1)=M(1,4,2 n+1)$ and $N(2,4,2 n)=$ $M(1,4,2 n)$.
\end{abstract}

\section{INTRODUCTION}

Let

$$
\pi=\pi_{0}+\pi_{1}+\cdots+\pi_{s-1}
$$

be a partition with the parts arranged in nonincreasing order. Dyson [D1] defined the rank of $\pi$ to be the number: $\pi_{0}-s$ (the empty partition of 0 has rank 0$)$. Writing $N(r, m, n)$ for the number of partitions of $n$ whose ranks are congruent to $r$ modulo $m$, he noticed that several relations appeared to hold between the numbers $N(r, m, k n+s)$ when $m=k=5$ and when $m=k=7$. Of particular interest are the relations

$$
N(0,5,5 n+4)=N(1,5,5 n+4)=\cdots=N(4,5,5 n+4)
$$

and

$$
N(0,7,7 n+5)=N(1,7,7 n+5)=\cdots=N(6,7,7 n+6),
$$

since they flesh out the Ramanujan congruences $[R]$, viz. $p(5 n+4) \equiv 0 \bmod 5$ and $p(7 n+5) \equiv 0 \bmod 7$. All Dyson's observations were shown to be correct by Atkin and Swinnerton-Dyer [ASD].

Ramanujan also showed that $p(11 n+6) \equiv 0 \bmod 11$. However, the values of their ranks mod 11 do not sort the partitions of $11 n+6$ into equal piles, and Dyson suggested there might exist a crank which would perform this trick. Such a crank was found by Andrews and Garvan [AG]. Here I give the (slightly different) crank of Dyson [D2]. With $t:=\pi_{0}-\pi_{1}$, the crank of $\pi$ is

$$
\begin{cases}t-\pi_{t}, & \text { if } t>0 \\ -s, & \text { if } t=0\end{cases}
$$

Received by the editors February 12, 1990 and, in revised form, June 5, 1990.

1980 Mathematics Subject Classification (1985 Revision). Primary 11P76.

Key words and phrases. Partition, rank, crank. 
Write $M(r, m, n)$ for the number of partitions of $n$ whose cranks are congruent to $r$ modulo $m$. Then

$$
M(0,11,11 n+6)=M(1,11,11 n+6)=\cdots=M(10,11,11 n+6) .
$$

Also, (1) and (2) hold (with $M$ for $N$ ) and there are numerous other relations between the $M(r, m, k n+s)$, when $(m, k)=(5,5),(7,7)$ and $(11,11)$ [G1] and when $(m, k)=(8,2),(9,3)$ and $(10,5)$ [G2]. Furthermore, there are several relations between the $M$ 's and the $N$ 's for $(m, k)=(5,5)$ and $(7,7)$ and several conjectural relations for $(m, k)=(8,4),(9,3)$ and $(12,2)$ [L]. I show here

Theorem. For all $n \geq 0$,

$$
\begin{aligned}
N(2,4,2 n) & =M(1,4,2 n), \\
N(0,4,2 n+1) & =M(1,4,2 n+1) .
\end{aligned}
$$

In what follows, $q$ denotes a complex number of modulus less than 1 , and $z$ is a nonzero complex number. Also

$$
\begin{aligned}
&(z)_{\infty}=(z ; q)_{\infty}:=(1-z)(1-z q)\left(1-z q^{2}\right) \cdots, \\
& {[z ; q]_{\infty}:=(z ; q)_{\infty}\left(z^{-1} q ; q\right)_{\infty} . }
\end{aligned}
$$

Note that

$$
[z q ; q]_{\infty}=\left[z^{-1} ; q\right]_{\infty}=-z^{-1}[z ; q]_{\infty}
$$

Also,

$$
P:=(q)_{\infty}^{-1}=\sum p(n) q^{n}
$$

where $p(n)$ is the number of partitions of $n$. Here, and below, $\sum_{\text {, denotes }}$ a sum over all integers (but note that $p(n)=0$ for $n<0$ ) and $\sum^{\prime}$ denotes a sum over all nonzero integers. If $X$ is a power series in $q, X_{\text {ev }}$ and $X_{\text {odd }}$ denote the power series in $q^{2}$ with

$$
X=X_{\mathrm{ev}}+X_{\mathrm{odd}} q .
$$

The techniques I use are closely modelled on those of Atkin and SwinnertonDyer [ASD]. They described their methods as old-fashioned, so mine are doubly so.

\section{LEMMAS}

Jacobi's triple product identity [A, Theorem 2.8 ] states that

$$
(q)_{\infty}[z ; q]_{\infty}=\sum(-)^{k} z^{k} q^{k(k-1) / 2} .
$$

Writing $q^{3}$ for $q$ and $q$ for $z$ in (5) gives Euler's identity [A, Corollary 1.7]:

$$
P^{-1}=(q)_{\infty}=\sum(-)^{k} q^{k(3 k-1) / 2} .
$$


Another identity I shall use is the quintuple product identity [Go]:

$$
(q)_{\infty}[z ; q]_{\infty}\left[z^{2} q ; q^{2}\right]_{\infty}=\left(q^{3} ; q^{3}\right)_{\infty}\left(\left[-z^{3} q ; q^{3}\right]_{\infty}-z\left[-z^{3} q^{2} ; q^{3}\right]_{\infty}\right) \text {. }
$$

Sorting the right-hand side of (6) into even and odd powers of $q$ and appealing to (5) and (7) gives

$$
(q)_{\infty}=\left(q^{16} ; q^{16}\right)_{\infty}\left(q^{2} ; q^{4}\right)_{\infty}\left(\left[-q^{6} ; q^{16}\right]_{\infty}-\left[-q^{2} ; q^{16}\right]_{\infty} q\right)
$$

Multiplying (8) by the result of replacing $q$ by $-q$ in (8) shows that

$$
\left[-q^{6} ; q^{16}\right]_{\infty}^{2}-\left[-q^{2} ; q^{16}\right]_{\infty}^{2} q^{2}=\left(q^{2} ; q^{4}\right)_{\infty}\left[q^{4} ; q^{8}\right]_{\infty}\left[q^{8} ; q^{16}\right]_{\infty}
$$

and from (8) and (9) we have

$$
P=\frac{\left(q^{16} ; q^{16}\right)_{\infty}}{\left(q^{2} ; q^{2}\right)_{\infty}^{2}}\left(\left[-q^{6} ; q^{16}\right]_{\infty}+\left[-q^{2} ; q^{16}\right]_{\infty} q\right)
$$

\section{Lemma 1.}

$$
\left[-q^{2} ; q^{4}\right]_{\infty}^{2}=\left[q ; q^{2}\right]_{\infty}^{2}\left[q^{2} ; q^{4}\right]_{\infty}+\left[-q^{4} ; q^{4}\right]_{\infty}^{2} q
$$

Proof. From (5), we have

$$
\left[-q ; q^{2}\right]_{\infty}=\left(\left[-q^{4} ; q^{8}\right]_{\infty}+\left[-q^{8} ; q^{8}\right]_{\infty} q\right)\left(q^{2} ; q^{4}\right)_{\infty}^{-1}\left(q^{4} ; q^{8}\right)_{\infty}^{-1}
$$

Set

From (12),

$$
f(q)=\left[-q^{2} ; q^{4}\right]_{\infty}^{2}-\left[q ; q^{2}\right]_{\infty}^{2}\left[q^{2} ; q^{4}\right]_{\infty}-\left[-q^{4} ; q^{4}\right]_{\infty}^{2} q
$$

$$
\begin{aligned}
f(-q)= & {\left[-q^{2} ; q^{4}\right]_{\infty}^{2}+\left[-q^{4} ; q^{4}\right]_{\infty}^{2} q } \\
& -\left(\left[-q^{4} ; q^{8}\right]_{\infty}+\left[-q^{8} ; q^{8}\right]_{\infty} q\right)^{2}\left[q^{4} ; q^{8}\right]_{\infty}^{-1} \\
= & {\left[q^{4} ; q^{8}\right]_{\infty}^{-1}\left(\left[-q^{2} ; q^{4}\right]_{\infty}^{2}\left[q^{4} ; q^{8}\right]_{\infty}-\left[-q^{4} ; q^{8}\right]_{\infty}^{2}-\left[-q^{8} ; q^{8}\right]_{\infty}^{2} q^{2}\right) } \\
= & -\left[q^{4} ; q^{8}\right]_{\infty}^{-1} f\left(-q^{2}\right) .
\end{aligned}
$$

Since $f(-q)$ has zero constant term, it is identically zero. So $f(q)$ is zero and (11) follows.

Now define (for any complex $\zeta$ )

$$
T(z, \zeta, q):=\sum(-)^{n} \frac{\zeta^{n} q^{3 n(n+1) / 2}}{\left(1-z q^{n}\right)}
$$

$T(z, \zeta, q)$ is an analytic function of $z$ in every region $0<z_{1} \leq z \leq z_{2}$, except for simple poles where $z=q^{n}$. Also, let

$$
T_{1}(\zeta, q):=\sum^{\prime}(-)^{n} \frac{\zeta^{n} q^{3 n(n+1) / 2}}{\left(1-q^{n}\right)}
$$

and let

$$
g(z)=g(z, q):=z \frac{\left[z^{2} ; q\right]_{\infty}}{[z ; q]_{\infty}} T(z, 1, q)-z^{3} T\left(z^{2}, z^{3}, q\right)-T_{1}\left(z^{-3}, q\right) .
$$


Atkin and Swinnerton-Dyer [ASD, (5.1)] showed that

$$
\begin{aligned}
\zeta^{3} T\left(z \zeta, \zeta^{3}, q\right)+T\left(z \zeta^{-1}, \zeta^{-3}, q\right)= & \zeta \frac{\left[\zeta^{2} ; q\right]_{\infty}}{[\zeta ; q]_{\infty}} T(z, 1, q) \\
& +\frac{[\zeta ; q]_{\infty}\left[\zeta^{2} ; q\right]_{\infty}(q ; q)_{\infty}^{2}}{[z \zeta ; q]_{\infty}[z ; q]_{\infty}\left[z \zeta^{-1} ; q\right]_{\infty}}
\end{aligned}
$$

that [ASD, (5.11)]

$$
g(z)-g(z q)=-3
$$

and that [ASD, (5.7)]

$$
g(z)+g\left(z^{-1} q\right)=1 .
$$

They also gave an expression for $2 g(z)-g\left(z^{2}\right)$ in terms of theta functions, but I need

\section{Lemma 2.}

$$
3 g(z)-g\left(z^{3}\right)=\frac{\left[z^{4} ; q\right]_{\infty}^{3}(q ; q)_{\infty}^{2}}{\left[z^{2} ; q\right]_{\infty}^{3}\left[z^{6} ; q\right]_{\infty}}+\frac{\left[z^{2} ; q\right]_{\infty}^{3}(q ; q)_{\infty}^{2}}{[z ; q]_{\infty}^{3}\left[z^{3} ; q\right]_{\infty}}-2
$$

Proof.

Let

$$
H(z):=\frac{\left[z^{2} ; q\right]_{\infty}^{3}(q ; q)_{\infty}^{2}}{[z ; q]_{\infty}^{3}\left[z^{3} ; q\right]_{\infty}}
$$

By (14), $3 g(z)-g\left(z^{3}\right)$ is unchanged under $z \mapsto z q$, as are $H(z)$ and $H\left(z^{2}\right)$ (by (4)). $H(z)$ and $H\left(z^{2}\right)$ together eliminate the poles of $3 g(z)-g\left(z^{3}\right)$ and so

$$
3 g(z)-g\left(z^{3}\right)-H(z)-H\left(z^{2}\right)=c,
$$

where $c$ is a constant [ASD, Lemma 2]. To determine $c$, note that, from (14) and (15),

$$
g(z)+g\left(z^{-1}\right)=-2 \text {. }
$$

Since

$$
H(-1)=0 \text { and } H(-i)=-H(i) \text {, }
$$

adding the results of setting $z=i$ and $z=-i$ in (17) and using (18) gives $c=-2$.

\section{THE PROOF}

Let $N(m, n)$ (respectively $M(m, n)$ ) denote the number of partitions of $n$ with rank (respectively crank) $m$. Then [D1, (20)]

$$
\sum \sum N(m, n) z^{m} q^{n}=\frac{(1-z)}{(q)_{\infty}} \sum \frac{(-)^{n} q^{n(3 n+1) / 2}}{\left(1-z q^{n}\right)} .
$$


(Note that, since we suppose the empty partition to have rank $0, N(0,0)=1$ in contrast to Dyson [D1] and Atkin and Swinnerton-Dyer [ASD], for whom $N(0,0)=0$.)

For integer $a$, let

$$
S(a):=\sum^{\prime} \frac{(-)^{n} q^{n(3 n+1) / 2+a n}}{\left(1-q^{4 n}\right)}
$$

Setting $z=i$ in (19), we find that

$$
A:=\sum(N(0,4, n)-N(2,4, n)) q^{n}=P(2 S(0)+2 S(1)+1)
$$

(note that $N(r, m, n)=N(m-r, m, n)$ ), and setting $z=-1$ in (19) gives

$$
B:=\sum(N(0,2, n)-N(1,2, n)) q^{n}=P(4 S(0)-4 S(1)+1) .
$$

Also $[\mathrm{AG}]$,

$$
\sum \sum M(m, n) z^{m} q^{n}=q-z^{-1} q+\frac{(q)_{\infty}}{(z q)_{\infty}\left(z^{-1} q\right)_{\infty}}
$$

and so (setting $z=-1$ in (21))

$$
\sum(M(0,2, n)-M(1,2, n)) q^{n}=2 q+C,
$$

where

$$
C:=\frac{(q)_{\infty}}{(-q)_{\infty}^{2}}
$$

Since $P=\sum(N(0,2, n)+N(1,2, n)) q^{n}$,

$$
\begin{aligned}
& P+B-2 A=4 \sum N(2,4, n) q^{n}, \\
& P+B+2 A=4 \sum N(0,4, n) q^{n} .
\end{aligned}
$$

Also, $P=\sum(M(0,2, n)+M(1,2, n)) q^{n}$ and (22) gives

$$
P-2 q-C=2 \sum M(1,2, n) q^{n}=-2 q+4 \sum M(1,4, n) q^{n}
$$

(since $M(1,2, n)=M(1,4, n)+M(3,4, n)$, and, for $n>1, M(1,4, n)=$ $M(3,4, n))$ and so

$$
P-C=4 \sum M(1,4, n) q^{n} .
$$

It follows from $(23 a)-(23 c)$ that the theorem will be proved once we show that

$$
(2 A-B)_{\mathrm{ev}}=C_{\mathrm{ev}}
$$

and

$$
(2 A+B)_{\text {odd }}=-C_{\text {odd }} .
$$

First we find expressions for $C_{\mathrm{ev}}$ and $C_{\mathrm{odd}}$. From (8) and (20c) we have

$$
C=\frac{(q)_{\infty}^{3}}{\left(q^{2} ; q^{2}\right)_{\infty}^{2}}=\frac{\left(q^{16} ; q^{16}\right)_{\infty}^{3}\left(q^{2} ; q^{4}\right)_{\infty}^{3}}{\left(q^{2} ; q^{2}\right)_{\infty}^{2}}\left(\left[-q^{6} ; q^{16}\right]_{\infty}-\left[-q^{2} ; q^{16}\right]_{\infty} q\right)^{3}
$$


and so

(25a)

$$
C_{\mathrm{ev}}=\frac{\left(q^{16} ; q^{16}\right)_{\infty}^{3}\left(q^{2} ; q^{4}\right)_{\infty}^{3}}{\left(q^{2} ; q^{2}\right)_{\infty}^{2}}\left(\left[-q^{6} ; q^{16}\right]_{\infty}^{3}+3\left[-q^{6} ; q^{16}\right]_{\infty}\left[-q^{2} ; q^{16}\right]_{\infty}^{2} q^{2}\right)
$$

and

(25b)

$$
C_{\text {odd }}=-\frac{\left(q^{16} ; q^{16}\right)_{\infty}^{3}\left(q^{2} ; q^{4}\right)_{\infty}^{3}}{\left(q^{2} ; q^{2}\right)_{\infty}^{2}}\left(3\left[-q^{6} ; q^{16}\right]_{\infty}^{2}\left[-q^{2} ; q^{16}\right]_{\infty}+\left[-q^{2} ; q^{16}\right]_{\infty}^{3} q^{2}\right)
$$

Next we find expressions for $(2 A-B)_{\mathrm{ev}}$ and $(2 A+B)_{\mathrm{odd}} \cdot(20 \mathrm{a})$ and $(20 \mathrm{~b})$ give

$$
2 A-B=P(8 S(1)+1)
$$

and

$$
2 A+B=P(8 S(0)+3)=8+P(8 S(4)-5)
$$

(because $S(0)-S(4)=\sum^{\prime}(-)^{k} q^{k(3 k-1) / 2}=P^{-1}-1$ (see (6)).

Straightforward manipulation gives

$$
\begin{aligned}
S(1)= & T_{1}\left(-q^{-18}, q^{16}\right)-q^{18} T\left(q^{12},-q^{18}, q^{16}\right) \\
& +\left(q^{6} T\left(q^{8},-q^{6}, q^{16}\right)-T\left(q^{4},-q^{-6}, q^{16}\right)\right) q^{3}
\end{aligned}
$$

and

$$
\begin{aligned}
S(4)= & T_{1}\left(-q^{-6}, q^{16}\right)-q^{6} T\left(q^{4},-q^{6}, q^{16}\right) \\
& +\left(q^{18} T\left(q^{8},-q^{18}, q^{16}\right)-T\left(q^{-4},-q^{-18}, q^{16}\right)\right) q^{-3} .
\end{aligned}
$$

Now (10), (13), (26a), (26b), (27a), and (27b) thrown together give

$$
(2 A-B)_{\mathrm{ev}}=\frac{\left(q^{16} ; q^{16}\right)_{\infty}}{\left(q^{2} ; q^{2}\right)_{\infty}^{2}}\left(\left[-q^{6} ; q^{16}\right]_{\infty}\left(1-8 g\left(-q^{6}\right)\right)\right.
$$

$$
\left.-8 \frac{\left[-q^{2} ; q^{16}\right]_{\infty}^{2}\left(q^{16} ; q^{16}\right)_{\infty}^{2}}{\left[-q^{6} ; q^{16}\right]_{\infty}\left[q^{8} ; q^{16}\right]_{\infty}} q^{4}\right)
$$

and

(28b)

$$
\begin{aligned}
(2 A+B)_{\text {odd }}=\frac{\left(q^{16} ; q^{16}\right)_{\infty}}{\left(q^{2} ; q^{2}\right)_{\infty}^{2}}\left(\left[-q^{2} ; q^{16}\right]_{\infty}\left(-5-8 g\left(-q^{2}\right)\right)\right. & \\
& \left.+8 \frac{\left[-q^{6} ; q^{16}\right]_{\infty}^{2}\left(q^{16} ; q^{16}\right)_{\infty}^{2}}{\left[-q^{2} ; q^{16}\right]_{\infty}\left[q^{8} ; q^{16}\right]_{\infty}}\right) .
\end{aligned}
$$

From (15) (with $z=-q^{6}$ and with $q^{16}$ for $q$ ) we have

$$
\begin{aligned}
& 3 g\left(-q^{6}\right)-g\left(-q^{18}\right) \\
& \quad=\left(-\frac{\left[q^{8} ; q^{16}\right]_{\infty}^{3}}{\left[q^{4} ; q^{16}\right]_{\infty}^{4}}+\frac{\left[q^{4} ; q^{16}\right]_{\infty}^{3}}{\left[-q^{6} ; q^{16}\right]_{\infty}^{3}\left[-q^{2} ; q^{16}\right]_{\infty}} q^{2}\right)\left(q^{16} ; q^{16}\right)_{\infty}^{2}-2
\end{aligned}
$$


and (with $z=-q^{2}$ )

$$
\begin{aligned}
& 3 g\left(-q^{2}\right)-g\left(-q^{6}\right) \\
& \quad=\left(\frac{\left[q^{8} ; q^{16}\right]_{\infty}^{3}}{\left[q^{4} ; q^{16}\right]_{\infty}^{4}}+\frac{\left[q^{4} ; q^{16}\right]_{\infty}^{3}}{\left[-q^{2} ; q^{16}\right]_{\infty}^{3}\left[-q^{6} ; q^{16}\right]_{\infty}}\right)\left(q^{16} ; q^{16}\right)_{\infty}^{2}-2 .
\end{aligned}
$$

From (14), (29), and (30) we find that

$$
\begin{aligned}
1-8 g\left(-q^{6}\right)=( & 2 \frac{\left[q^{8} ; q^{16}\right]_{\infty}^{3}}{\left[q^{4} ; q^{16}\right]_{\infty}^{4}}-\frac{\left[q^{4} ; q^{16}\right]_{\infty}^{3}}{\left(-q^{2} ; q^{4}\right)_{\infty}^{3}} \\
& \left.\times\left(\left[-q^{6} ; q^{16}\right]_{\infty}^{2}+3\left[-q^{2} ; q^{16}\right]_{\infty}^{2} q^{2}\right)\right)\left(q^{16} ; q^{16}\right)_{\infty}^{2}
\end{aligned}
$$

and

$$
-5-8 g\left(-q^{2}\right)=\left(-2 \frac{\left[q^{8} ; q^{16}\right]_{\infty}^{3}}{\left[q^{4} ; q^{16}\right]_{\infty}^{4}}-\frac{\left[q^{4} ; q^{16}\right]_{\infty}^{3}}{\left(-q^{2} ; q^{4}\right)_{\infty}^{3}}\right.
$$

$$
\left.\times\left(3\left[-q^{6} ; q^{16}\right]_{\infty}^{2}+\left[-q^{2} ; q^{16}\right]_{\infty}^{2} q^{2}\right)\right)\left(q^{16} ; q^{16}\right)^{2}
$$

Inserting (31a), (31b) into (28a), (28b) (note that $\left[q^{8} ; q^{16}\right]_{\infty}\left[q^{4} ; q^{16}\right]_{\infty}^{-2}=$ $\left.\left[-q^{4} ; q^{8}\right]_{\infty}\right)$ gives

$$
\begin{aligned}
(2 A-B)_{\mathrm{ev}}= & \frac{\left(q^{16} ; q^{16}\right)_{\infty}^{3}}{\left(q^{2} ; q^{2}\right)_{\infty}^{2}}\left[-q^{6} ; q^{16}\right]_{\infty} \\
& \times\left(2\left[q^{8} ; q^{16}\right]_{\infty}\left[-q^{4} ; q^{8}\right]_{\infty}^{2}-\left(\left[-q^{6} ; q^{16}\right]_{\infty}^{2}\right.\right.
\end{aligned}
$$

$$
\left.\left.+3\left[-q^{2} ; q^{16}\right]_{\infty}^{2} q^{2}\right)\left(q^{2} ; q^{4}\right)_{\infty}^{3}-8 \frac{\left[-q^{2} ; q^{16}\right]_{\infty}^{2}}{\left[-q^{6} ; q^{16}\right]_{\infty}^{2}\left[q^{8} ; q^{16}\right]_{\infty}} q^{4}\right)
$$

and

$$
\begin{aligned}
(2 A+B)_{\text {odd }}= & \frac{\left(q^{16} ; q^{16}\right)_{\infty}^{3}}{\left(q^{2} ; q^{2}\right)_{\infty}^{2}}\left[-q^{2} ; q^{16}\right]_{\infty} \\
& \times\left(-2\left[q^{8} ; q^{16}\right]_{\infty}\left[-q^{4} ; q^{8}\right]_{\infty}^{2}-\left(3\left[-q^{6} ; q^{16}\right]_{\infty}^{2}\right.\right. \\
& \left.\left.\quad+\left[-q^{2} ; q^{16}\right]_{\infty}^{2} q^{2}\right)\left(q^{2} ; q^{4}\right)_{\infty}^{3}+8 \frac{\left[-q^{6} ; q^{16}\right]_{\infty}^{2}}{\left[-q^{2} ; q^{16}\right]_{\infty}^{2}\left[q^{8} ; q^{16}\right]_{\infty}}\right) .
\end{aligned}
$$

If we now compare (32a), (32b) with (25a), (25b), we see that (24a) (and so also (3a)) holds if

$$
\begin{aligned}
X:= & {\left[q^{8} ; q^{16}\right]_{\infty}\left[-q^{4} ; q^{8}\right]_{\infty}^{2}-\left(\left[-q^{6} ; q^{16}\right]_{\infty}^{2}+3\left[-q^{2} ; q^{16}\right]_{\infty}^{2} q^{2}\right)\left(q^{2} ; q^{4}\right)_{\infty}^{3} } \\
& -4 \frac{\left[-q^{2} ; q^{16}\right]_{\infty}^{2}}{\left[-q^{6} ; q^{16}\right]_{\infty}^{2}\left[q^{8} ; q^{16}\right]_{\infty}} q^{4}=0
\end{aligned}
$$


and that $(24 b)$ and $(3 b)$ hold if

$$
\begin{aligned}
Y:= & {\left[q^{8} ; q^{16}\right]_{\infty}\left[-q^{4} ; q^{8}\right]_{\infty}^{2}+\left(3\left[-q^{6} ; q^{16}\right]_{\infty}^{2}+\left[-q^{2} ; q^{16}\right]_{\infty}^{2} q^{2}\right)\left(q^{2} ; q^{4}\right)_{\infty}^{3} } \\
& -4 \frac{\left[-q^{6} ; q^{16}\right]_{\infty}^{2}}{\left[-q^{2} ; q^{16}\right]_{\infty}^{2}\left[q^{8} ; q^{16}\right]_{\infty}}=0 .
\end{aligned}
$$

But

$$
\begin{aligned}
Y-X= & 4\left(\left[-q^{6} ; q^{16}\right]_{\infty}^{2}+\left[-q^{2} ; q^{16}\right]_{\infty}^{2} q^{2}\right) \\
& \times\left(\left(q^{2} ; q^{4}\right)_{\infty}^{3}-\frac{1}{\left[-q^{2} ; q^{4}\right]_{\infty}\left[q^{8} ; q^{16}\right]_{\infty}}\left(\left[-q^{6} ; q^{16}\right]_{\infty}^{2}-\left[-q^{2} ; q^{16}\right]_{\infty}^{2} q^{2}\right)\right) \\
= & 0
\end{aligned}
$$

by (9), and

$$
\begin{aligned}
& Y+X=2\left(\left[q^{8} ; q^{16}\right]_{\infty}\left[-q^{4} ; q^{8}\right]_{\infty}^{2}+\left(\left[-q^{6} ; q^{16}\right]_{\infty}^{2}-\left[-q^{2} ; q^{16}\right]_{\infty}^{2} q^{2}\right)\left(q^{2} ; q^{4}\right)_{\infty}^{3}\right. \\
&\left.-\frac{2}{\left[-q^{2} ; q^{4}\right]_{\infty}\left[q^{8} ; q^{16}\right]_{\infty}}\left(\left[-q^{6} ; q^{16}\right]_{\infty}^{4}+\left[-q^{2} ; q^{16}\right]_{\infty}^{4} q^{4}\right)\right) \\
&=2\left[q^{8} ; q^{16}\right]_{\infty}\left(\left[-q^{4} ; q^{8}\right]_{\infty}^{2}-\left[q^{2} ; q^{4}\right]_{\infty}^{2}\left[q^{4} ; q^{8}\right]_{\infty}-\left[-q^{8} ; q^{8}\right]_{\infty}^{2} q^{2}\right)
\end{aligned}
$$

(using (9))

$$
=0 \text {, }
$$

by (11). So $X=Y=0$ and the theorem is proved.

\section{SOME QUESTIONS}

In the following list, the "=" have been established by Garvan [G2] and the " =" " follow from the theorem proved above. The " $\stackrel{?}{=}$ " are guesses that I cannot prove [L].

$$
\begin{aligned}
& M(1,8,4 n)=M(3,8,4 n){ }^{*} N(2,8,4 n) \stackrel{?}{=} N(4,8,4 n), \\
& M(2,8,4 n) \stackrel{?}{=} N(3,8,4 n) . \\
& M(0,8,4 n+1)+M(1,8,4 n+1) \stackrel{\stackrel{?}{=}}{=} N(1,8,8,4 n+1)+M(4,8,4 n+1) \\
& \stackrel{?}{=} N(3,8,4 n+1)+N(4,8,4 n+1), \\
& M(2,8,4 n+1) \stackrel{?}{=} N(3,8,4 n+1) . \\
& M(1,8,4 n+2)=M(3,8,4 n+2) \\
&={ }^{*} N(2,8,4 n+2) \stackrel{?}{=} N(0,8,4 n+2), \\
& M(2,8,4 n+2) \stackrel{?}{=} N(1,8,4 n+2) .
\end{aligned}
$$




$$
\begin{aligned}
M(0,8,4 n+3)+M(1,8,4 n+3) & =M(3,8,4 n+3)+M(4,8,4 n+3) \\
& \stackrel{?}{=} N(0,8,4 n+3)+N(1,8,4 n+3) \\
& \stackrel{?}{=} N(2,8,4 n+3)+N(3,8,4 n+3), \\
M(2,8,4 n+3) & \stackrel{?}{=} N(1,8,4 n+3) .
\end{aligned}
$$

Using the techniques of this paper, I can reduce the " $\stackrel{?}{=}$ " to certain "identities" between theta functions. I have considerable computer evidence for these "identities" but, as yet, I am unable to nail them down.

Note. Nicolas Santa-Gadea (thesis, Pennsylvania State University) has found proofs of all the " $\stackrel{?}{=}$ " of the last section. He uses the third order mock theta functions. I also have proofs using the classical theory of modular forms.

\section{REFERENCES}

[A] George E. Andrews, The theory of partitions, Encyclopedia of Mathematics 2, AddisonWesley, Reading, 1976.

[AG] George.E. Andrews and F. G. Garvan, Dyson's crank of a partition, Bull. Amer. Math. Soc. 18 (1988), 166-171.

[ASD] A. O. L. Atkin and P. Swinnerton-Dyer, Some properties of partitions, Proc. London Math. Soc. (3) 4 (1954), 84-106.

[D1] F. J. Dyson, Some guesses in the theory of partitions, Eureka 8 (1944), 10-15.

[D2] _ - Mappings and symmetries of partitions, J. Combin. Theory Ser. A 51 (1989), 169-180.

[G1] F. G. Garvan, New combinatorial interpretations of Ramanujan's partition congruences mod 5, 7 and 11, Trans. Amer. Math. Soc. 305 (1988), 47-77.

[G2] - The crank of partitions mod 8, 9 and 10, Trans. Amer. Math. Soc. (to appear).

[Go] B. Gordon, Some identities in combinatorial analysis, Quart. J. Math. Oxford Ser. (2) 12 (1961), 285-290.

[L] Richard Lewis, On some relations between the rank and the crank, J. Combin. Theory Ser. A (to appear).

[R] S. Ramanujan, Some properties of $p(n)$, the number of partitions of $n$, Paper 25 of Collected Papers of S. Ramanujan, Cambridge University Press, London and New York, 1927; reprinted Chelsea, New York, 1962.

Mathematics Division, University of Sussex, Falmer, Brighton, Sussex, BN1 9QH, UNITED KINGDOM 